\title{
OPEN Ascorbic acid during the suckling period is required for proper DNA demethylation in the liver
}

\author{
Kenichi Kawahori ${ }^{1}$, Yoshitaka Kondo ${ }^{2,3}$, Xunmei Yuan $^{1,4}{ }^{4}$ Yuki Kawasaki $^{5}$, Nozomi Hanzawa ${ }^{1}$, \\ Kazutaka Tsujimoto ${ }^{1}$, Fumiko Wada ${ }^{1}$, Takashi Kohda ${ }^{5,6}$, Akihito Ishigami ${ }^{2}$, Tetsuya Yamada ${ }^{1}$, \\ Yoshihiro Ogawa ${ }^{4,7 凶}$ \& Koshi Hashimoto ${ }^{8,9 \square}$
}

Ascorbic acid (AA, vitamin C) serves as a cofactor for ten-eleven translocation (TET) enzymes and induces DNA demethylation in vitro. However, its role in DNA demethylation in vivo remains unclear. We previously reported that DNA demethylation in the mouse liver was enhanced during the suckling period. Therefore, we hypothesized that DNA demethylation is enhanced in an AA-dependent manner during the suckling period. To examine our hypothesis, we employed wild-type (WT) mice, which synthesize AA, and senescence marker protein-30/gluconolactonase (SMP30/GNL) knockout (KO) mice, which cannot synthesize AA, and analyzed the DNA methylation status in the livers of offspring in both the suckling period and adulthood. SMP30/GNL KO offspring showed DNA hypermethylation in the liver possibly due to low plasma and hepatic AA levels during the suckling period despite the administration of rescue-dose AA to dams. Furthermore, DNA hypermethylation of the fibroblast growth factor 21 gene (Fgf21), a PPAR $\alpha$ target gene, persisted into adulthood. In contrast, a high-dose AA administration to SMP30/GNL KO dams during the lactation period restored DNA demethylation in the livers of offspring. Even though a slight increase was observed in plasma AA levels with the administration of rescue-dose AA to WT dams during the gestation and lactation periods, DNA demethylation in the livers of offspring was minimally enhanced. The present results demonstrate that AA intake during the suckling period is required for proper DNA demethylation in the liver.

Ten-eleven translocation (TET) enzymes catalyze the oxidation of 5-methylcytosine ( $5 \mathrm{mC}$ ) to 5-hydroxymethylcytosine $(5 \mathrm{hmC})^{1-3}$. Ascorbic acid (AA, vitamin C) promoted DNA demethylation in embryonic stem cells and induced pluripotent stem cells ${ }^{4,5}$ and was also shown to enhance $5 \mathrm{hmC}$ levels in a TET-dependent manner ${ }^{6-8}$. Recent studies on humans suggested that plasma AA concentrations are related to aberrant DNA methylation in genomic DNA derived from peripheral leukocytes in patients with some cancers ${ }^{9,10}$. However, it currently remains unclear whether AA affects DNA methylation patterns in vivo.

Humans, other primates, and animal species, such as Guinea pigs and fruit bats, lack the enzyme L-gulonolactone oxidase, which catalyzes the final step in AA biosynthesis; therefore, they are incapable of synthesizing $\mathrm{AA}^{11}$. Senescence marker protein-30 (SMP30) is involved in AA biosynthesis in non-primate mammals ${ }^{12,13}$. We previously reported that SMP30 served as a gluconolactonase (GNL), which catalyzed the penultimate step of

\footnotetext{
${ }^{1}$ Department of Molecular Endocrinology and Metabolism, Graduate School of Medical and Dental Sciences, Graduate School of Medical and Dental Sciences, Tokyo Medical and Dental University, Tokyo 113-8510, Japan. ${ }^{2}$ Molecular Regulation of Aging, Tokyo Metropolitan Institute of Gerontology, Tokyo 173-0015, Japan. ${ }^{3}$ Biomedical Gerontology Laboratory, Faculty of Human Sciences, Waseda University, Tokorozawa 359-1192, Japan. ${ }^{4}$ Department of Molecular and Cellular Metabolism, Graduate School of Medical and Dental Sciences, Tokyo Medical and Dental University, Tokyo 113-8510, Japan. ${ }^{5}$ Department of Epigenetics, Medical Research Institute, Tokyo Medical and Dental University, Bunkyo-ku, Tokyo 113-8510, Japan. ${ }^{6}$ Laboratory of Embryology and Genomics, Department of Biotechnology, Faculty of Life and Environmental Sciences, University of Yamanashi, Kofu, Yamanashi 400-8510, Japan. ${ }^{7}$ Department of Medicine and Bioregulatory Science, Graduate School of Medical Sciences, Kyushu University, 3-1-1 Maidashi, Higashi-ku, Fukuoka 812-8582, Japan. ${ }^{8}$ Department of Preemptive Medicine and Metabolism, Graduate School of Medical and Dental Sciences, Tokyo Medical and Dental University, 1-5-45 Yushima, Bunkyo-ku, Tokyo 113-8510, Japan. ${ }^{9}$ Department of Diabetes, Endocrinology and Hematology, Dokkyo Medical University Saitama Medical Center, 2-1-50 Minami-Koshigaya, Koshigaya, Saitama 343-8555, Japan. ${ }^{\boxplus}$ email: yogawa@med.kyushu-u.ac.jp; k-hashi@ dokkyomed.ac.jp
} 
the AA biosynthesis pathway ${ }^{13,14}$. Therefore, SMP30/GNL-deficient (knockout: KO) mice fed an AA-free diet exhibited the symptoms of scurvy. Moreover, an insufficient AA intake during the gestation period induced dilated cardiomyopathy in perinatal SMP30/GNL KO mice ${ }^{15}$. Therefore, SMP30/GNL KO mice are suitable for investigating the physiological functions of AA in vivo.

Despite the importance of AA in development in childhood ${ }^{15-18}$, the molecular mechanisms by which AA affects development remain unclear. We previously reported the marked DNA demethylation of the fibroblast growth factor 21 gene $(F g f 21)$, a peroxisome proliferator-activated receptor (PPAR) a target gene, in the liver during the suckling period ${ }^{19}$. In recent years, AA has been shown to affect DNA demethylation in vitro, whereas its effects in vivo are still unknown.

In the present study, we focused on the potential role of AA in DNA demethylation in the liver. We investigated whether AA affects DNA demethylation in the mouse liver during the suckling period. Using a genomewide analysis of DNA methylation, we identified genes susceptible to AA-dependent DNA demethylation in the liver during the suckling period. We also demonstrated that the DNA methylation status of Fgf21, a PPARa target gene, in early life was maintained in later life.

\section{Results}

SMP30/GNL KO mice exhibited DNA hypermethylation in livers of offspring due to low plasma and hepatic AA levels during the suckling period. SMP30/GNL KO mice, which cannot synthesize AA, were crossed to obtain SMP30/GNL KO offspring. We previously reported that SMP30/GNL KO offspring were born and matured normally using free access to water containing $1.5 \mathrm{~g} / \mathrm{L} \mathrm{AA}$, defined as a rescue dose to avoid the effects of an AA deficiency ${ }^{20}$, for dams and offspring ${ }^{14}$. Therefore, we administered $1.5 \mathrm{~g} / \mathrm{L} \mathrm{AA}$ water to SMP30/GNL KO dams during the gestation and lactation periods. In a preliminary experiment, we assessed AA levels in milk obtained from the gastric contents of offspring 16 days after birth (d16), which was derived from AA supplemented and unsupplemented KO dams during the lactation period (Supplementary Fig. 1a). We found that AA levels, including AA and dehydroascorbic acid (DHA), the oxidized form of AA, in milk from supplemented $\mathrm{KO}$ dams were significantly higher than in that from unsupplemented dams (Supplementary Fig. 1b). These results suggested that the administration of $1.5 \mathrm{~g} / \mathrm{L}$ AA water to SMP30/GNL KO dams during the lactation period significantly increased AA levels in milk.

Upon weaning, we administered AA-free water and $1.5 \mathrm{~g} / \mathrm{L}$ AA water to wild-type (WT) (N) and SMP30/ GNL KO (KO) offspring, respectively (Fig. 1a). We examined $\mathrm{N}$ and $\mathrm{KO}$ offspring in both the suckling period (d16) and in adulthood (d100). As shown in Fig. 1b, no significant differences were observed in plasma or milk AA levels between $\mathrm{N}$ and $\mathrm{KO}$ dams upon delivery (d2). The body weights of offspring in both groups were similar from birth to adulthood (Fig. 1c). Moreover, the liver weights of offspring in both groups were similar on d16 and d100 (Supplementary Fig. 2a). However, the plasma and hepatic AA levels on d16 were significantly higher in $\mathrm{N}$ offspring than in $\mathrm{KO}$ offspring (Fig. 1d, left panel). On the other hand, in adulthood (d100), no significant differences were noted in plasma or hepatic AA levels between $\mathrm{N}$ and $\mathrm{KO}$ offspring (Fig. 1d, right panel). Collectively, these results indicated that AA levels were significantly lower in KO offspring than in $\mathrm{N}$ offspring predominantly during the suckling period.

We also employed a Microarray-based Integrated Analysis of Methylation by Isoschizomers (MIAMI) ${ }^{21}$ to examine the genome-wide DNA methylation status of the livers of $\mathrm{N}$ and $\mathrm{KO}$ offspring. On d16, KO offspring had more hypermethylated genes than $\mathrm{N}$ offspring, which was also confirmed on d100. More than 2000 genes were hypermethylated in KO offspring relative to those in N offspring on both d16 and d100 (Table 1a, Supplementary Table 1a-d). These results suggested that AA affected DNA demethylation in the liver during the suckling period, and that the administration of $1.5 \mathrm{~g} / \mathrm{L}$ AA water to $\mathrm{KO}$ dams was insufficient to induce physiological levels of DNA demethylation in the livers of KO offspring. A pathway analysis of these genes yielded cell proliferation- and differentiation-related pathways and glucose- or lipid metabolism-related pathways (Table 1b). We identified 437 genes that were hypermethylated in KO offspring relative to those in $\mathrm{N}$ offspring on both d 16 and d 100 (Fig. 2, Supplementary Table 2). We also found 9 PPAR a target genes that were hypermethylated on both $\mathrm{d} 16$ and $\mathrm{d} 100$ (Table 2), and a pathway analysis of the 437 genes confirmed that the fatty acid metabolism pathway was significant, which included Fgf21 (Fig. 2, Table 2), a gene that was reportedly subjected to PPARadependent DNA demethylation during the suckling period ${ }^{19,22}$.

We previously identified $F g f 21$ as an epigenetic memory gene that plays a pivotal role in the developmental programming of obesity ${ }^{22}$. In silico searches revealed 21 sites for CpG dinucleotides (i.e., cytosine followed by guanine) for DNA methylation around the transcription start site (TSS) of Fgf21 (Fig. 3a) ${ }^{22,23}$. To verify whether Fgf21 DNA demethylation physiologically occurred in an AA-dependent manner, we examined the DNA methylation status of the $F g f 21$ promoter region in the livers of $\mathrm{N}$ and $\mathrm{KO}$ offspring using a bisulfite-sequencing analysis. The results of the bisulfite-sequencing analysis revealed that the difference in the Fgf21 DNA methylation status on d16 between $\mathrm{N}$ and $\mathrm{KO}$ offspring remained until d100 (Fig. 3b). We also evaluated the DNA methylation status at each CpG site in the Fgf21 promoter region on d2, d16, and d100 and found some CpG sites at which the DNA methylation rate in KO offspring was significantly higher than that in $\mathrm{N}$ offspring (Supplementary Fig. 3). However, no specific regulatory elements were detected at these CpG sites.

On the other hand, DNA demethylation did not increase lipid metabolism-related gene expression, including Fgf21, in the livers of $\mathrm{N}$ offspring at a steady state (Fig. 3c). Accordingly, no significant differences were observed in serum triglyceride (TG), total cholesterol (T-Chol), non-esterified fatty acids (NEFA), or blood glucose levels between $\mathrm{N}$ and $\mathrm{KO}$ offspring on d100 (Supplementary Fig. 2b).

Administration of high-dose AA to SMP30/GNL KO dams during the lactation period restored DNA demethylation in livers of offspring. Based on the results obtained, we administered high-dose 
a
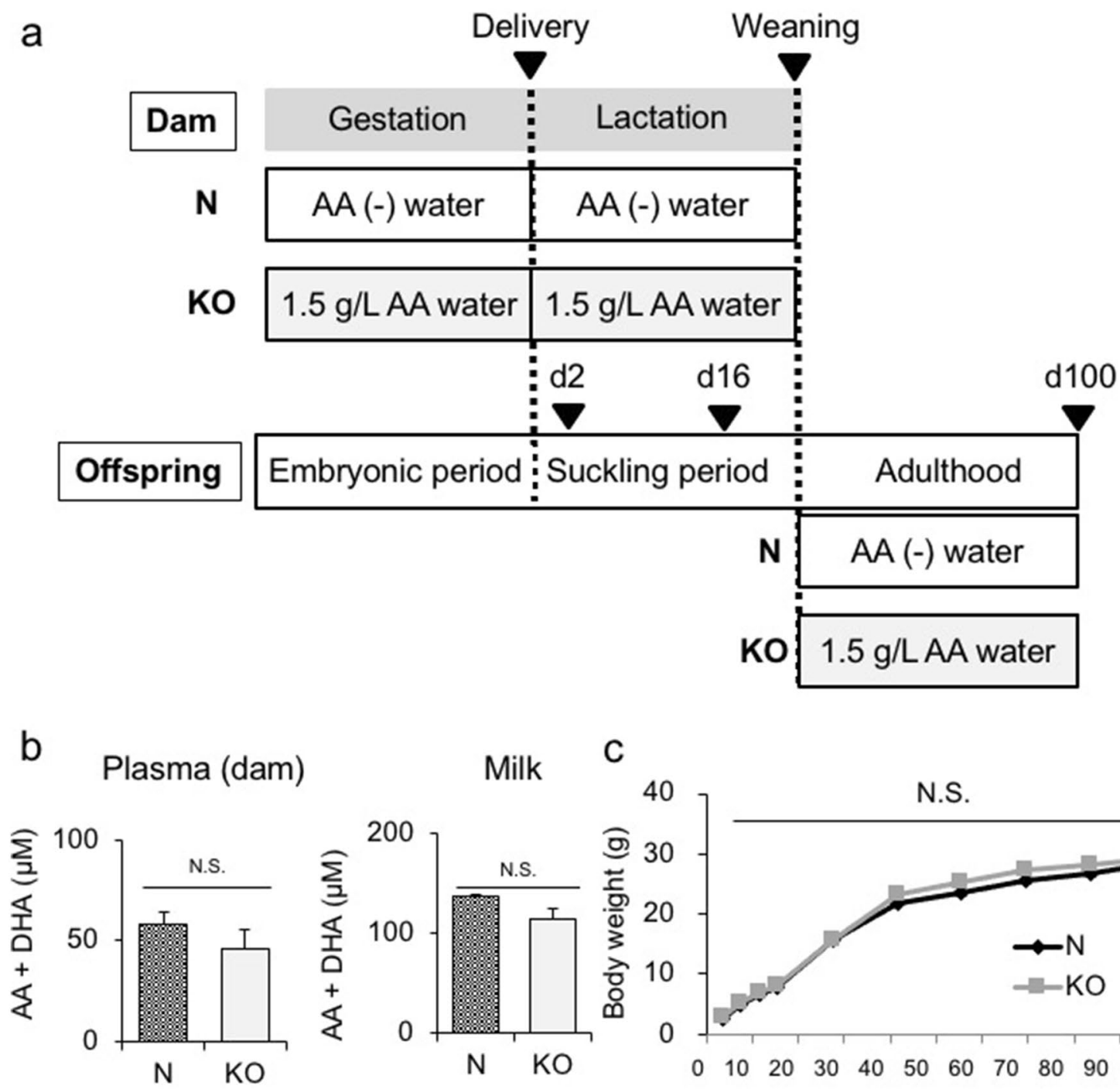

C 40

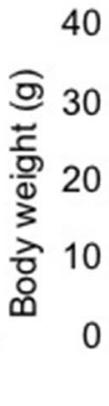

N.S.

d

d16
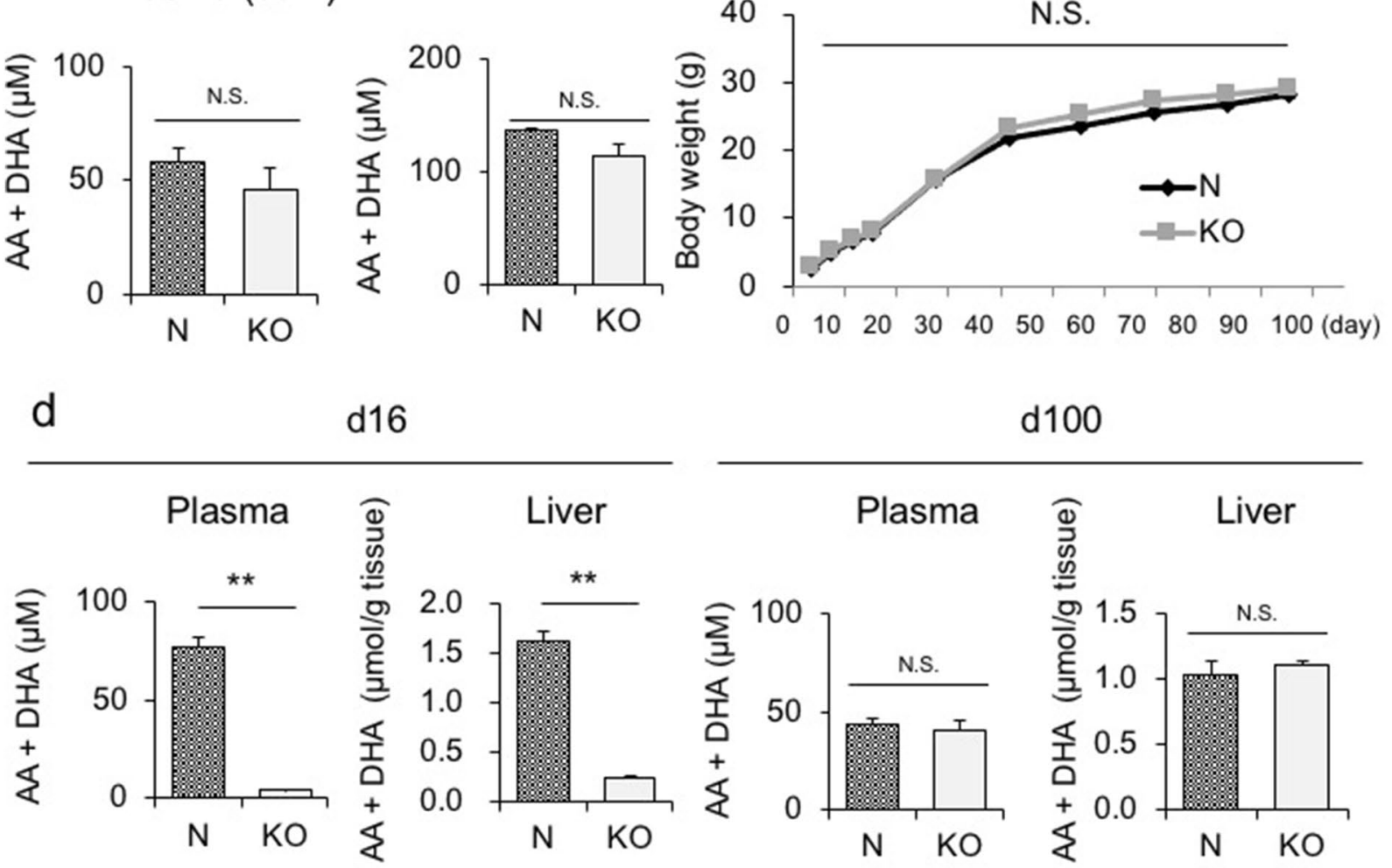

Figure 1. Analysis of SMP30/GNL KO offspring. (a) Experimental protocol to analyze WT (N) and SMP30/ GNL KO (KO) offspring. Free access to water containing $1.5 \mathrm{~g} / \mathrm{L}$ AA was given to SMP30/GNL KO dams and offspring throughout the experiment, whereas $\mathrm{N}$ dams and offspring were fed AA-free water. (b) Plasma and milk AA levels of dams in the KO and $\mathrm{N}$ groups on $\mathrm{d} 2$ ( $\mathrm{n}=6-8$ per group). (c) Body weights of $\mathrm{KO}$ and $\mathrm{N}$ offspring from birth to $\mathrm{d} 100$ ( $\mathrm{n}=6$ per group, statistical analysis using a two-way ANOVA with Tukey's multiple comparison test). (d) Plasma and hepatic AA levels in $\mathrm{KO}$ and $\mathrm{N}$ offspring on d16 (left panel) and d100 (right panel) ( $n=6$ per group). Statistical analysis using an unpaired Student's $t$-test unless otherwise indicated. Data are expressed as the mean \pm SEM. ${ }^{* *} p<0.01$; N.S., not significant vs. KO offspring. 


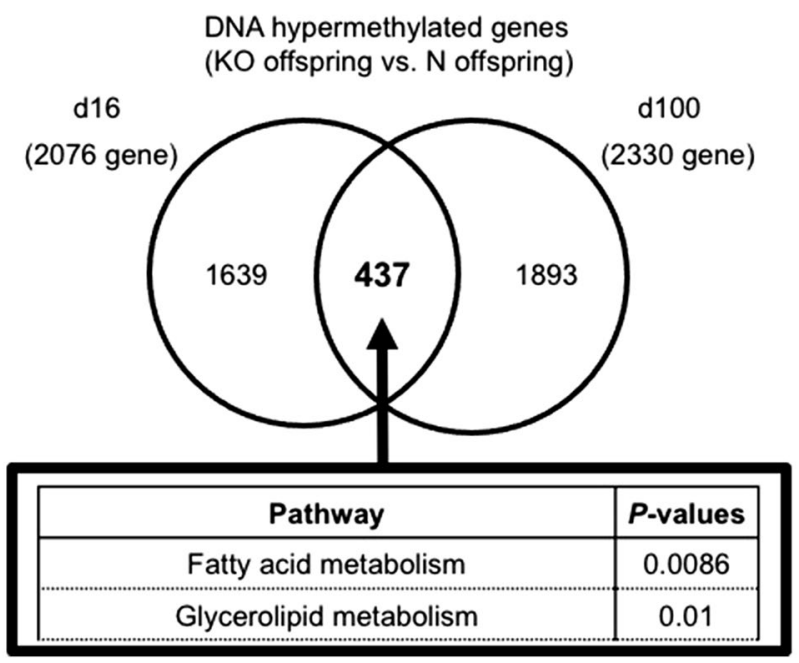

Figure 2. Venn diagram showing results of MIAMI. (a) We identified 437 genes that were DNA hypermethylated in $\mathrm{KO}$ offspring relative to those in $\mathrm{N}$ offspring on both $\mathrm{d} 16$ and d100. We performed a pathway analysis with these genes.

\begin{tabular}{|c|c|c|c|}
\hline & \multicolumn{3}{|l|}{ Number of genes } \\
\hline & Total number DNA & DNA hypomethylation & DNA hypermethylation \\
\hline \multicolumn{4}{|l|}{ (a) } \\
\hline d16 & 27,110 & 361 & 2076 \\
\hline d100 & 28,347 & 574 & 2330 \\
\hline \multicolumn{4}{|l|}{ (b) d16 } \\
\hline Pathway & $P$ values & & \\
\hline Glycerolipid metabolism & $3.8 \mathrm{E}-4$ & & \\
\hline mTOR signaling pathway & $4.7 \mathrm{E}-3$ & & \\
\hline Insulin signaling pathway & $5.4 \mathrm{E}-3$ & & \\
\hline ErbB signaling pathway & $5.6 \mathrm{E}-3$ & & \\
\hline Adherens junction & $1.0 \mathrm{E}-2$ & & \\
\hline Neurotrophin signaling pathway & $1.2 \mathrm{E}-2$ & & \\
\hline Axon guidance & $1.3 \mathrm{E}-2$ & & \\
\hline PPAR signaling pathway & $1.4 \mathrm{E}-2$ & & \\
\hline Glycine, serine and threonine metabolism & $1.5 \mathrm{E}-2$ & & \\
\hline Fatty acid metabolism & $3.2 \mathrm{E}-2$ & & \\
\hline \multicolumn{4}{|l|}{ (c) d100 } \\
\hline Pathway & $P$ values & & \\
\hline Wnt signaling pathway & $2.3 \mathrm{E}-5$ & & \\
\hline Pathway in cancer & $2.6 \mathrm{E}-5$ & & \\
\hline Basal cell carcinoma & $3.6 \mathrm{E}-5$ & & \\
\hline TGF-beta signaling pathway & $5.1 \mathrm{E}-5$ & & \\
\hline Insulin signaling pathway & $2.8 \mathrm{E}-3$ & & \\
\hline Neurotrophin signaling pathway & $2.2 \mathrm{E}-2$ & & \\
\hline Glycosphingolipid biosynthesis & $2.3 \mathrm{E}-2$ & & \\
\hline MAPK signaling pathway & $3.1 \mathrm{E}-2$ & & \\
\hline mTOR signaling pathway & $3.1 \mathrm{E}-2$ & & \\
\hline
\end{tabular}

Table 1. (a) The number of genes with DNA methylation changes in the liver of KO offspring relative to those in $\mathrm{N}$ offspring on $\mathrm{d} 16$ and d100. (b) Pathway analysis of genes that were DNA hypermethylated in KO offspring relative to those in $\mathrm{N}$ offspring on $\mathrm{d} 16$ (top) and $\mathrm{d} 100$ (bottom). 


\begin{tabular}{|l|l|}
\hline Gene symbol & Gene name \\
\hline Cptla & carnitine palmitoyltransferase la, muscle \\
\hline Fgf21 & fibroblast growth factor 21 \\
\hline Acaala & acetyl-Coenzyme A acyltransferase 1A \\
\hline Ehhadh & enoyl-CoA hydratase and 3-hydroxyacyl CoA dehydrogenase \\
\hline Peci & penoyl-Coenzyme A delta isomerase 2 \\
\hline Aldh3a2 & aldehyde dehydrogenase family 3, subfamily A2 \\
\hline Agpat2 & 1-acylglycerol-3-phosphate O-acyltransferase 2 \\
\hline Srebfl & sterol regulatory element binding transcription factor 1 \\
\hline Pnpla2 & patatin-like phospholipase domain containing 2 \\
\hline
\end{tabular}

Table 2. PPARa target genes that were DNA hypermethylated on both d16 and d100 (KO offspring vs. N offspring).

AA to $\mathrm{KO}$ dams during the lactation period as a second experiment to clarify whether DNA demethylation in $\mathrm{KO}$ offspring was restored. Upon delivery (d2), KO dams were divided into the $\mathrm{KO}$ and KOA groups. The $\mathrm{KO}$ group had free access to $1.5 \mathrm{~g} / \mathrm{L}$ AA water, whereas the KOA group was given $3.0 \mathrm{~g} / \mathrm{L}$ AA water orally and injected intraperitoneally (i.p.) with $4.0 \mathrm{~g} / \mathrm{L} \mathrm{AA}$ (Fig. 4a). On d2, plasma AA levels were significantly higher in KOA dams than in KO dams (Fig. 4b). Body and liver weights in both groups were similar between $\mathrm{d} 4$ and d16 (Fig. 4c). However, plasma and hepatic AA levels were significantly higher in KOA offspring than in KO offspring on d16 (Fig. 4d).

To clarify whether DNA demethylation in KOA offspring was induced by increased TET catalysis, we analyzed $5 \mathrm{hmC}$ levels in the livers of $\mathrm{KOA}$ and $\mathrm{KO}$ offspring on d16. The results obtained showed that $5 \mathrm{hmC}$ levels in the liver were significantly higher in KOA offspring than in KO offspring (Fig. 4e). These results indicated that the activation of TET led to the DNA demethylation of genes in the livers of KOA offspring.

MIAMI of the offspring on d16 revealed that more genes were hypomethylated in KOA offspring than in KO offspring. In total, 716 and 172 genes were hypomethylated and hypermethylated, respectively, in KOA offspring relative to those in $\mathrm{KO}$ offspring, suggesting that the high-dose AA administration to KOA dams restored DNA demethylation in the livers of their offspring (Table 3a, Supplementary Table 3a, b). We performed a pathway analysis of the 716 genes that were hypomethylated in KOA offspring, which yielded Wnt, TGF- $\beta$, Jak-STAT, and insulin pathways with significant differences (Table 3b, Supplementary Table 3a). We also found 5 PPAR a target genes among the 716 genes (Table 3c). However, Fgf21 was not included among the 5 genes.

We identified 163 common genes between the 716 genes hypomethylated in KOA offspring and the 2076 genes hypermethylated in the KO offspring relative to those in wild-type (N) offspring on d16 (Table 1 and Supplementary Table $1 b$ ). These 163 genes were presumably hypermethylated in KO offspring relative to those in $\mathrm{N}$ offspring and hypomethylated by the high-dose AA supplementation (Supplementary Table 4).

We also performed MIAMI to compare the DNA methylation status in the livers of KOA and N offspring and identified 1034 genes that were hypermethylated in KOA offspring relative to those in $\mathrm{N}$ offspring on d16, similar to KO offspring (Supplementary Table 5a, 6a,b). A pathway analysis of the 1034 genes revealed cell proliferation and differentiation-related pathways and the glucose or lipid metabolism-related pathway, which was similar to KO offspring shown in Table 1 (Supplementary Table 5b).

Rescue-dose AA administration did not exert an additive effect on DNA demethylation in livers of WT mice. As described above, WT mice are capable of synthesizing AA, in contrast to humans and Guinea pigs. In the third experiment, we administered AA-free water and diet to WT dams until delivery. Upon delivery, during the lactation period, dams were divided into two groups: one was fed AA-free water and the other was fed $1.5 \mathrm{~g} / \mathrm{L}$ AA water. Male offspring of the dams fed AA-free water and $1.5 \mathrm{~g} / \mathrm{L}$ AA water were referred to as $\mathrm{N}$ and A offspring, respectively (Fig. 5a). On d16, the plasma and milk AA levels of dams were significantly higher in the A group than in the $\mathrm{N}$ group (Fig. 5b). Body and liver weights in both groups were similar from birth to d16 (Fig. 5c, d). On d16, plasma and hepatic AA levels were significantly higher in the A group than in the $\mathrm{N}$ group (Fig. 5e).

In addition, $5 \mathrm{hmC}$ levels in livers were not significantly different between the $\mathrm{N}$ and A groups. These results suggested that in the livers of WT mice, in which AA levels are sufficient, slight increases in AA levels did not induce DNA demethylation through TET activity (Fig. 5f).

We employed MIAMI to analyze the genome-wide DNA methylation status in the livers of offspring in both groups on d 16 (Table 4). We found that 560 and 409 genes were hypomethylated and hypermethylated, respectively, in the A group relative to those in the $\mathrm{N}$ group (Table 4, Supplementary Table 7a, b). MIAMI revealed that the number of hypomethylated genes was similar in the A and N groups, which confirmed that a slight increase in plasma and liver AA levels in WT mice had a minimal effect on DNA demethylation.

\section{Discussion}

In the present study to elucidate the role of AA in DNA demethylation in the murine liver during the suckling period, we performed three experiments using WT and SMP30/GNL KO mice.

We employed MIAMI to evaluate the DNA methylation status of genes in the liver. In MIAMI, we only evaluated the DNA methylation status on the HpaII/MspI restriction enzyme site CCGG. Therefore, we did 
a Fgf21 BS region

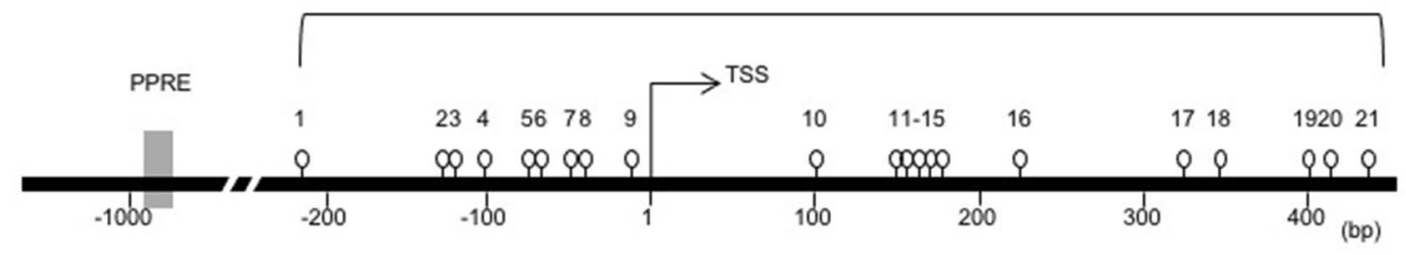

b

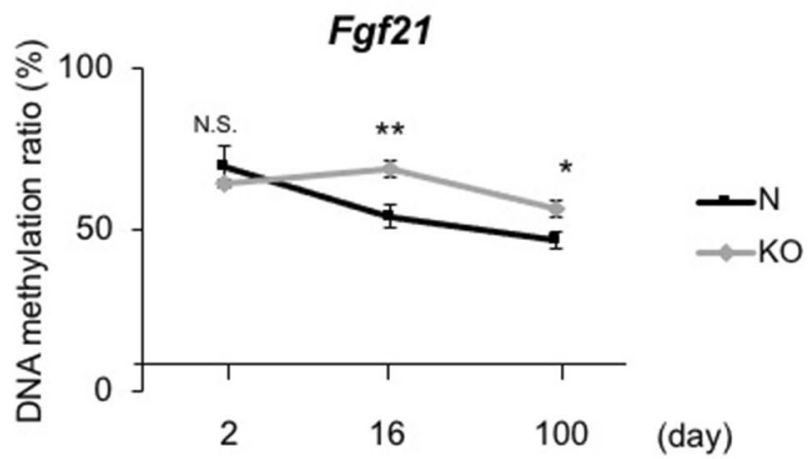

N
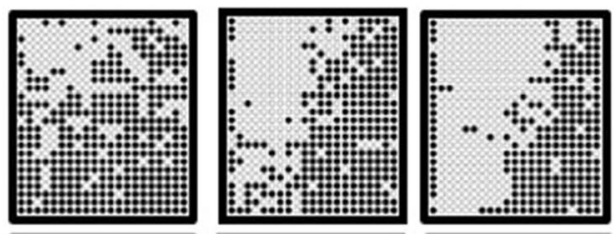

$\mathrm{KO}$
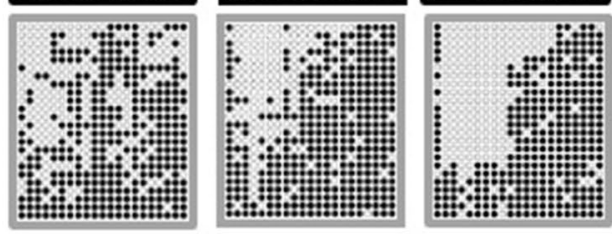

C

d100
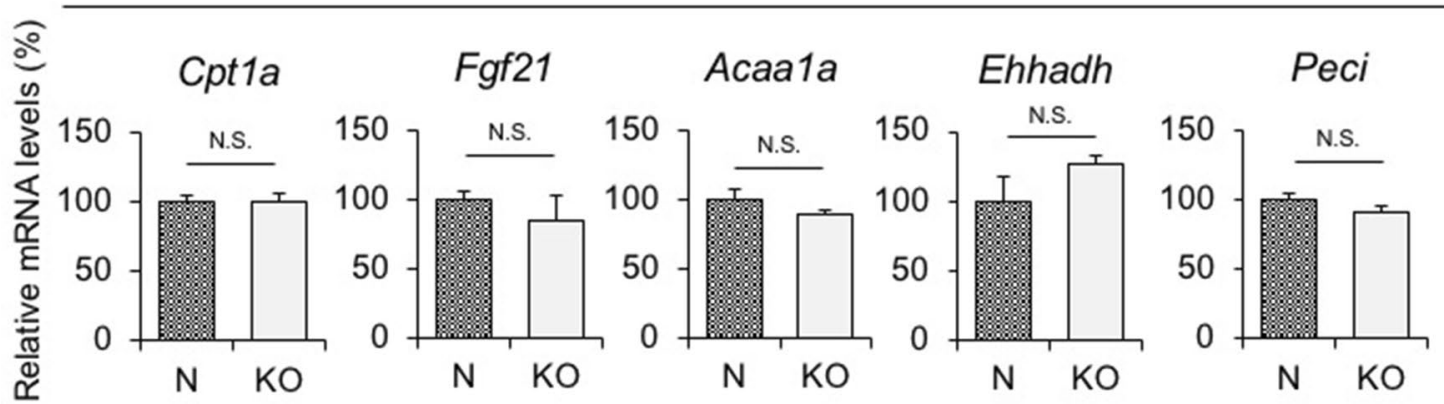

Figure 3. DNA methylation status and gene expression levels of PPARa target genes in livers of KO and N offspring. (a) Schematic representation of the promoter region of Fgf21. Open circles and gray boxes indicate CpG sites and PPAR response elements (PPREs), respectively. The bisulfite-sequencing (BS) analysis region encompassing the transcription start site (TSS) is indicated. (b) Fgf21 DNA methylation status in the livers of $\mathrm{N}$ and $\mathrm{KO}$ offspring via the BS analysis ( $\mathrm{n}=4$ per group, statistical analysis using a one-way ANOVA with Tukey's multiple comparison test). Representative BS data are depicted below the graph. Data are expressed as the mean \pm SEM. ${ }^{\star} p<0.05$; ${ }^{* *} p<0.01$; N.S., not significant vs. KO offspring. (c) Hepatic Cpt1a, Fgf21, Acaa1a, Ehhadh, and Peci mRNA expression levels on d16, which were DNA hypermethylated on both d16 and d100. $(\mathrm{n}=6$ per group). Statistical analysis using an unpaired Student's $t$-test unless otherwise indicated. Data are expressed as the mean \pm SEM. N.S., not significant vs. KO offspring. 


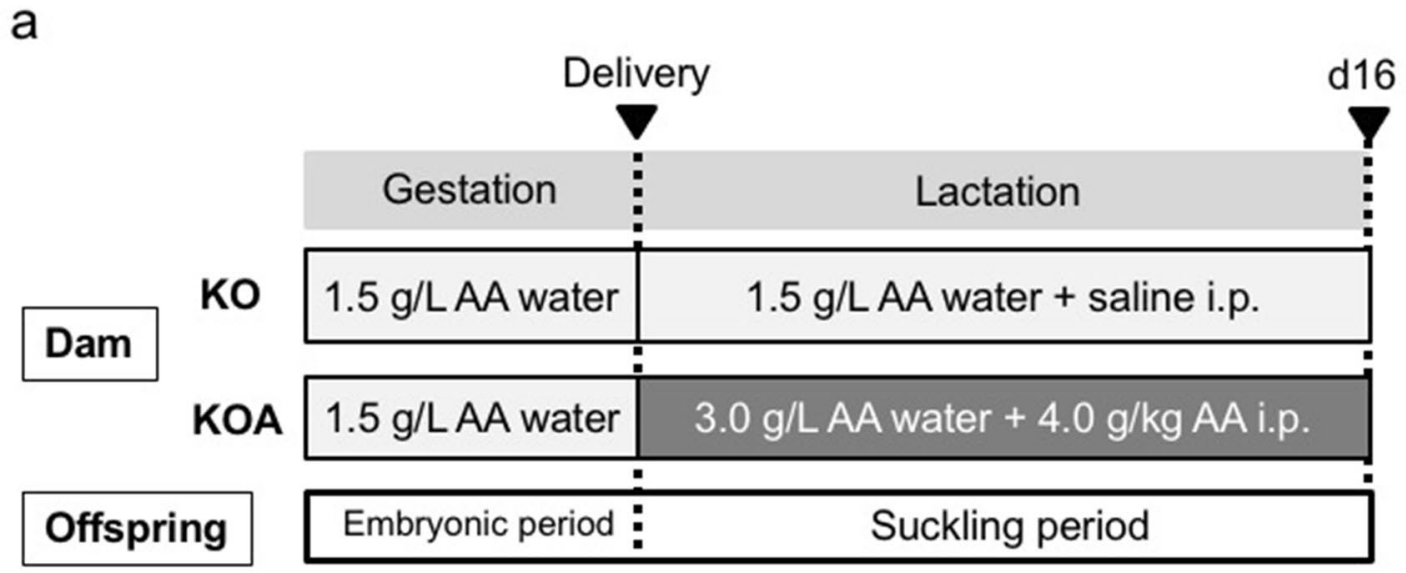

b

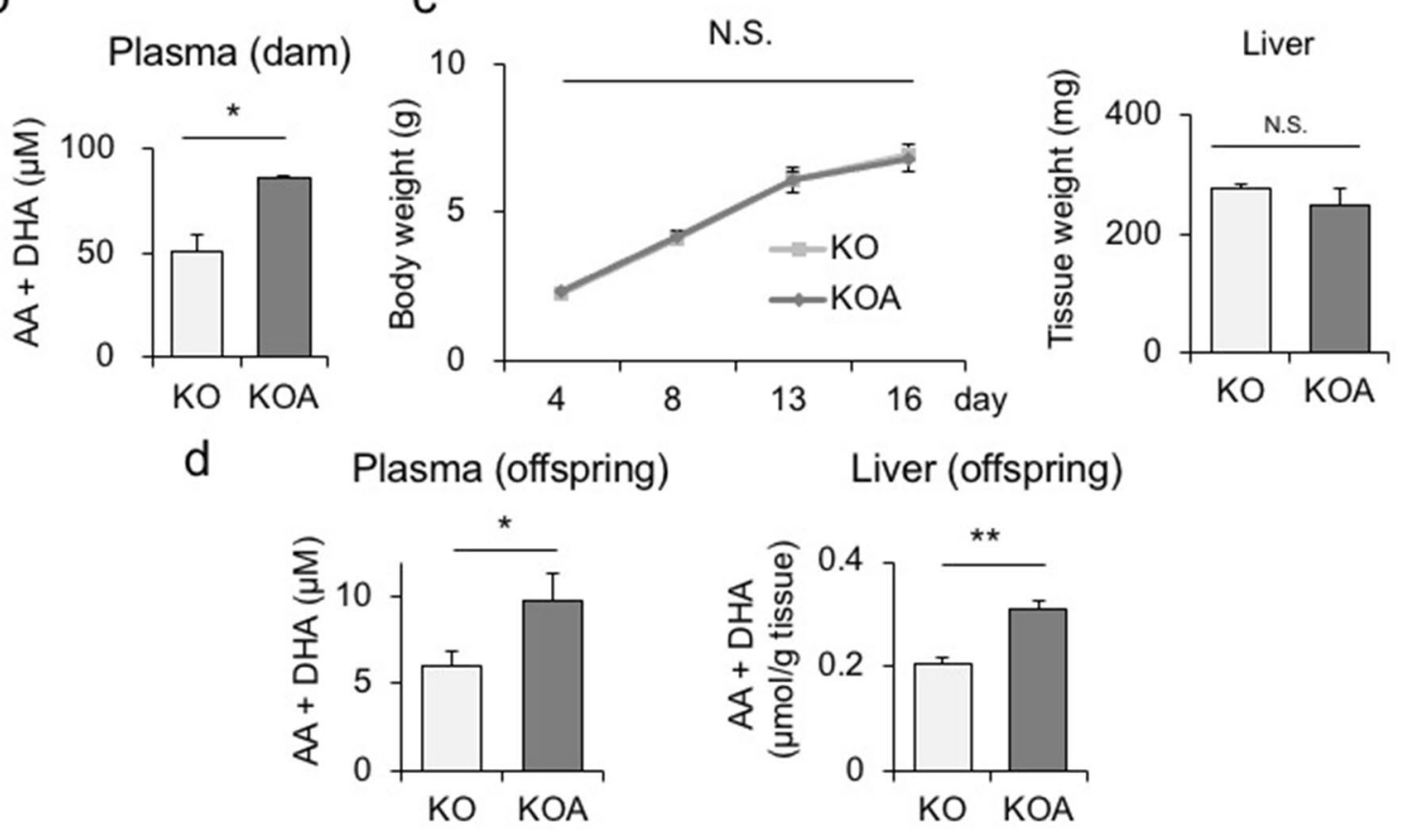

e

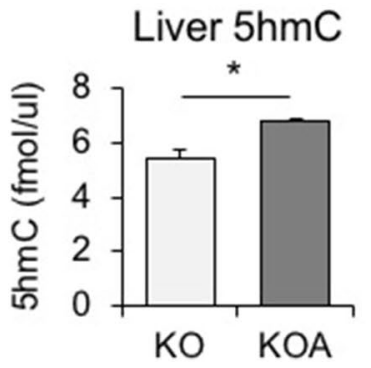

Figure 4. Maternal administration of high-dose AA to SMP30/GNL KO dams during the lactation period. (a) Experimental protocol of the maternal administration of high-dose AA to SMP30/GNL KO dams during the lactation period. Upon delivery (d2), KO dams were divided into the $\mathrm{KO}$ and $\mathrm{KOA}$ groups. The $\mathrm{KO}$ group had free access to $1.5 \mathrm{~g} / \mathrm{L} \mathrm{AA}$ water, whereas the KOA group was given $3.0 \mathrm{~g} / \mathrm{L} \mathrm{AA}$ water orally and injected intraperitoneally (i.p.) with $4.0 \mathrm{~g} / \mathrm{L}$ AA. Upon birth, during the lactation period, male offspring were divided into the two groups as above. Plasma AA levels in dams on $\mathrm{d} 2$ ( $\mathrm{n}=6$ per group). (c) Body weights of KO and KOA offspring from birth to d16 (left panel, statistical analysis using a one-way ANOVA with Tukey's multiple comparison test) and liver weight on $\mathrm{d} 16$ (right panel) ( $\mathrm{n}=6$ per group). (d) Plasma and hepatic AA levels in $\mathrm{KO}$ and KOA offspring on $\mathrm{d} 16$ ( $\mathrm{n}=6-8$ per group). (e) $5 \mathrm{hmC}$ levels in the livers of $\mathrm{KO}$ and $\mathrm{KOA}$ offspring on $\mathrm{d} 16$ ( $\mathrm{n}=6$ per group). Statistical analysis using an unpaired Student's $t$-test unless otherwise indicated. Data are expressed as the mean \pm SEM. ${ }^{*} p<0.05 ;{ }^{* *} p<0.01$; N.S., not significant vs. KOA offspring. 


\begin{tabular}{|c|c|c|c|}
\hline & \multicolumn{3}{|l|}{ Number of genes } \\
\hline & Total number & DNA hypomethylation & DNA hypermethylation \\
\hline (a) d16 & 29,307 & 716 & 172 \\
\hline \multicolumn{4}{|l|}{ (b) } \\
\hline Pathway & $P$ values & & \\
\hline Wnt signaling pathway & $2.5 \mathrm{E}-5$ & & \\
\hline Axon guidance & $3.2 \mathrm{E}-4$ & & \\
\hline TGF-beta signaling pathway & $7.3 \mathrm{E}-3$ & & \\
\hline Cell cycle & $8.9 \mathrm{E}-3$ & & \\
\hline Jak-STAT signaling pathway & $1.1 \mathrm{E}-2$ & & \\
\hline MAPK signaling pathway & $1.3 \mathrm{E}-2$ & & \\
\hline Insulin signaling pathway & $3.7 \mathrm{E}-2$ & & \\
\hline \multicolumn{4}{|l|}{ (c) } \\
\hline Gene symbol & Gene name & & \\
\hline Acot7 & acyl-CoA thioesterase 7 & & \\
\hline Abcg8 & ATP binding cassette subfamily G member 8 & & \\
\hline Scarb2 & Scavenger receptor class B, member 2 & & \\
\hline Srebf 1 & $\begin{array}{l}\text { Sterol regulatory element binding transcription } \\
\text { factor } 1\end{array}$ & & \\
\hline Ifi47 & Interferon gamma-inducible protein 47 & & \\
\hline
\end{tabular}

Table 3. (a) The number of genes with DNA methylation changes in the liver of KOA offspring relative to those in KO offspring on d16. (b) Pathway analysis of genes that were DNA hypomethylated in KOA offspring relative to those in $\mathrm{KO}$ offspring on d16. (c) PPAR target genes that were DNA hypomethylated on d16 (KOA offspring vs. KO offspring).

not examine whole DNA methylation sites, which is a limitation of the present study. Moreover, the DNA methylation status of each gene was calculated as a relative, not an absolute value. Therefore, we were unable to precisely observe changes in the DNA methylation status of each gene with AA supplementation, which is another limitation.

The AA transporters, sodium-dependent vitamin C transporter (SVCT) 1 and SVCT2, and DHA transporters, glucose transporter (GLUT) 1, GLUT3, and GLUT4, are expressed in the small intestine of both WT and SMP30/GNL KO mice ${ }^{24}$. Therefore, $\mathrm{N}$ and $\mathrm{KO}$ offspring are both capable of taking in AA and DHA from milk. In the first experiment, even though plasma and milk AA levels in SMP30/GNL KO dams were similar to those in WT dams, plasma and hepatic AA levels were markedly lower in KO offspring than in N offspring, suggesting that AA levels in offspring during the suckling period largely depended on the intrinsic synthesis of AA in the offspring themselves. Furthermore, markedly low AA levels contributed to global DNA hypermethylation in the liver. Therefore, the intrinsic synthesis of AA in offspring during the suckling period appears to be necessary and sufficient for proper DNA demethylation in the liver. Although we found no morphological changes in the livers of the offspring in any of the experiments conducted, $\mathrm{KO}$ offspring in later life (d100) exhibited the DNA hypermethylation of genes in the mTOR, Wnt, TGF- $\beta$, and MAPK signaling pathways, which are essential for organogenesis and development of the liver. Therefore, AA may exert DNA demethylation effects in many organs, including the liver, because a lack of AA in early life was shown to inhibit normal development of the brain, heart, and lungs ${ }^{15,25}$. Moreover, in a previous study ${ }^{15}$, we reported embryonic lethality and severe organ developmental disorders in SMP30/GNL KO mice without AA supplementation, which may be attributed to aberrant DNA methylation.

Although AA levels in KO offspring were restored by the administration of $1.5 \mathrm{~g} / \mathrm{L}$ AA water in adulthood, the DNA methylation status of PPARa target genes, including Fgf21, was hypermethylated relative to those in N offspring and persisted into adulthood, suggesting that the suckling period may be the 'critical window' for the modulation of the DNA methylation status in the liver, which we recently reported ${ }^{19,22}$. These results led to the second experiment to support this hypothesis because high-dose AA administration to KOA dams during the lactation period induced global DNA demethylation in the livers of KOA offspring duringthe suckling period.

AA induces TET-dependent DNA demethylation in vitro ${ }^{15,25}$. The present results supported AA also inducing DNA demethylation in vivo. Hypermethylated genes in $\mathrm{KO}$ offspring relative to those in $\mathrm{N}$ offspring on d16 and d100 included lipid metabolism-related genes, such as Fgf21, suggesting that an AA deficiency in the suckling period results in disorders in hepatic lipid metabolism in the offspring to adulthood. SMP30/GNL KO mice without AA supplementation developed fatty liver and dyslipidemia in adulthood ${ }^{25,26}$. DiTroia et al. recently attempted to elucidate the role of AA in the developing germline, employing Gulo knockout mice, which cannot synthesize their own $\mathrm{AA}^{27,28}$. They demonstrated that a maternal AA deficiency during gestation up to embryonic day 13.5 did not affect overall embryonic development, but led to reduced numbers of germ cells, delayed meiosis, and reduced fecundity in adult offspring. They also showed that it led to the aberrant DNA methylation of meiosis regulators, transposable elements, and genes related to lipid and body weight homeostasis in the embryonic germline of the progeny, which was observed in germ cells in TET1 knockout mice ${ }^{27,29}$. These findings 
a

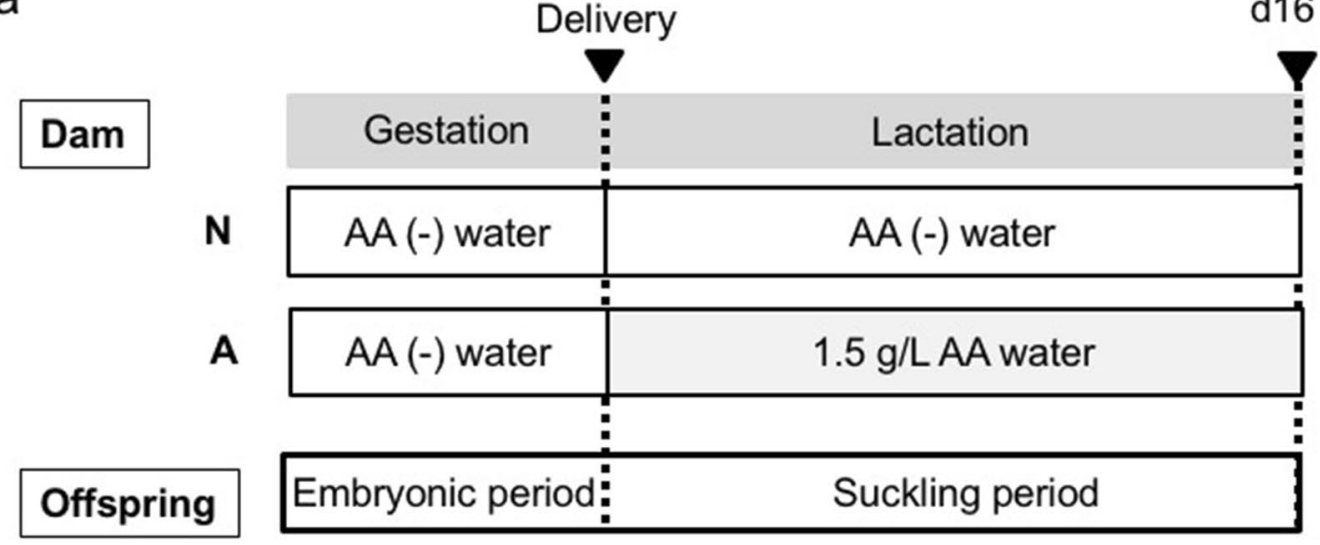

b
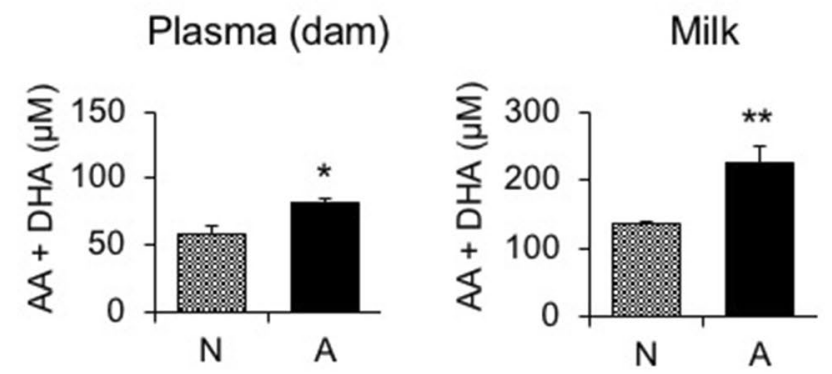

C

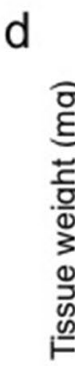

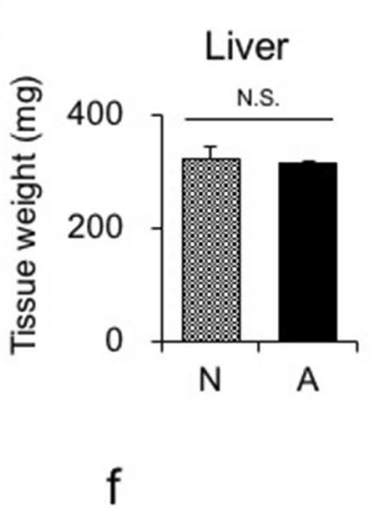

e
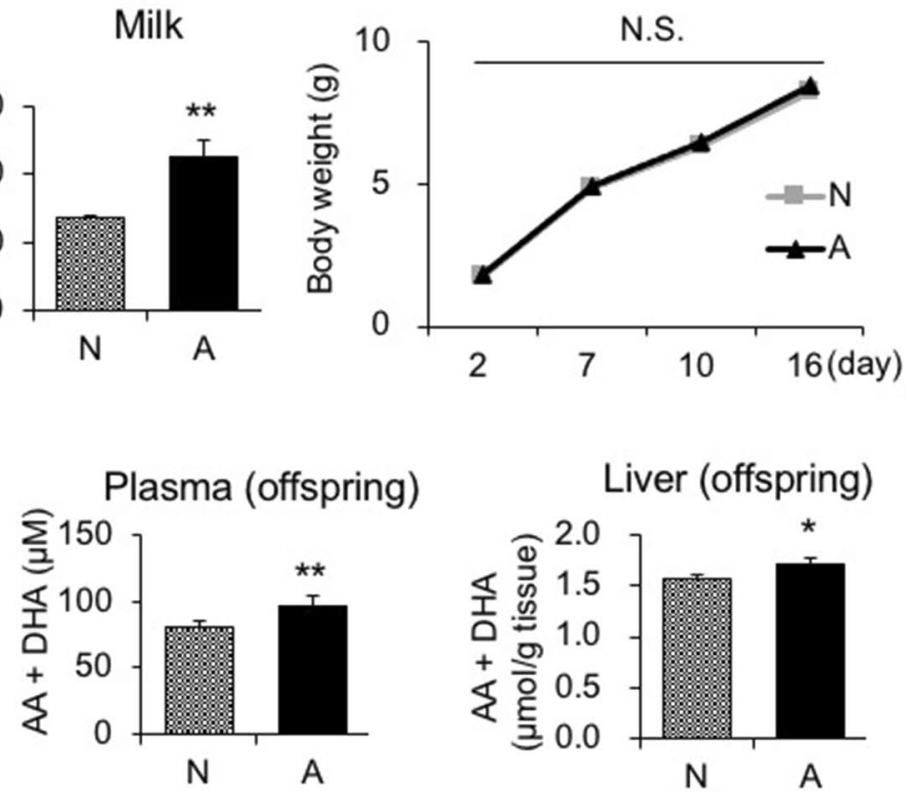

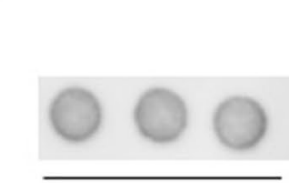

$\mathrm{N}$

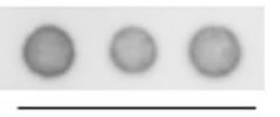

A

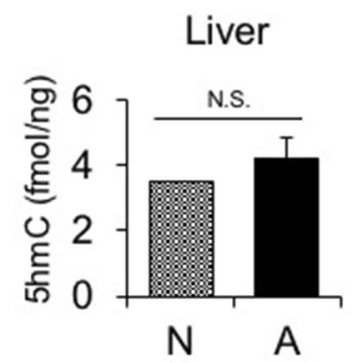

Figure 5. Maternal administration of rescue-dose AA to WT dams during the lactation period. (a) Experimental protocol of the maternal administration of AA to WT dams during the lactation period. Upon delivery (d2), dams were divided into two groups; one was fed AA-free water, referred to as the N group, and the other was fed $1.5 \mathrm{~g} / \mathrm{L}$ AA water as the A group. Upon birth, during the lactation period, male offspring were divided into the two groups as above. (b) Plasma AA levels in dams on d16 ( $\mathrm{n}=6-8$ per group). (c) Body weights of $\mathrm{N}$ and A offspring from birth to d16 (left panel, statistical analysis using a one-way ANOVA with Tukey's multiple comparison test) and (d) liver weight on d16 (right panel) ( $\mathrm{n}=6-8$ per group). (e) Plasma and hepatic AA levels in $\mathrm{N}$ and A offspring on d16 ( $\mathrm{n}=6-8$ per group). (f) $5 \mathrm{hmC}$ levels in the livers of $\mathrm{N}$ and A offspring on $\mathrm{d} 16$ ( $\mathrm{n}=3$ per group). Statistical analysis using an unpaired Student's $t$-test unless otherwise indicated. Data are expressed as the mean \pm SEM. ${ }^{\star} p<0.05 ;{ }^{* *} p<0.01$; N.S., not significant vs. A offspring. 


\begin{tabular}{|l|l|l|l|}
\hline \multirow{2}{*}{ d16 } & \multicolumn{2}{|l|}{ Number of genes } & DNA hypermethylation \\
\cline { 2 - 4 } & Total number & DNA hypomethylation & 409 \\
\hline
\end{tabular}

Table 4. The number of genes with DNA methylation changes in the liver of A offspring relative to those in $\mathrm{N}$ offspring on d 16 .

suggested that AA is required for proper DNA demethylation in germ cells and that lipid metabolism-related genes are susceptible to AA-dependent DNA demethylation during the gestation period, which is consistent with the present results.

In the first experiment, even though hepatic lipid metabolism-related genes were hypermethylated in KO offspring, no significant differences were observed in gene expression levels or serum lipid parameters between $\mathrm{N}$ and KO offspring. Previous studies reported that differences in the DNA methylation status may not reflect differences in steady-state gene expression ${ }^{30,31}$. However, in a transcriptionally active state, even subtle differences in the DNA methylation status may induce marked differences in gene expression ${ }^{30-32}$. Thus, some environmental cues, such as a high-fat diet, may be required to highlight changes in lipid metabolism-related gene expression and the metabolic phenotypes of KO offspring in adulthood. In the third experiment, the administration of $1.5 \mathrm{~g} / \mathrm{L} \mathrm{AA}$ water to WT dams resulted in a slight increase in plasma and hepatic AA levels in their offspring. However, this increase did not markedly induce any additional DNA demethylation in the liver, which suggested that the intrinsic synthesis of AA in the offspring was sufficient for proper DNA demethylation. Since humans are incapable of synthesizing AA, the present results are of significance; a maternal AA deficiency during the lactation period may induce the DNA hypermethylation of genes related to organogenesis and lipid metabolism in the livers of babies, which may result in organ disorders and metabolic diseases in adulthood. A previous study reported that AA prevented an aberrant DNA methylation status in offspring associated with maternal smoking in pregnancy ${ }^{18}$.

In the present study, we did not administer high-dose AA, which was administered to KOA dams, to WT dams. However, since KOA offspring had more hypermethylated genes than $\mathrm{N}$ offspring on d16, similar to KO offspring by MIAMI, high-dose AA administered to KOA dams was not sufficient to restore plasma AA levels in KOA offspring. Moreover, because Fgf21 was not included in the genes that were hypomethylated in KOA offspring relative to those in $\mathrm{KO}$ offspring on $\mathrm{d} 16$, it is conceivable that plasma AA levels in KOA offspring did not induce the hypomethylation of Fgf21.

We identified 163 genes that were presumably hypermethylated in $\mathrm{KO}$ offspring and hypomethylated by highdose AA supplementation (Supplementary Table 4). Based on these results, the few genes hypermethylated in KO offspring were hypomethylated by high-dose AA supplementation, which may have been attributed to the lower plasma AA concentration in KO offspring despite high-dose AA supplementation than in $\mathrm{N}$ offspring. However, we do not think that only a small fraction of genes hypermethylated in KO offspring were hypomethylated by high-dose AA supplementation because 172 genes were hypermethylated in KOA offspring relative to those in KO offspring on d16 (Table 3a), whereas up to 2076 genes were hypermethylated in KO offspring relative to those in $\mathrm{N}$ offspring (Table 1a). Further studies are needed to clarify whether the administration of markedly higher doses of AA to WT dams induces hypomethylation in the livers of their offspring, which was a limitation of the present study.

In conclusion, the present study provides in vivo evidence to show that AA epigenetically regulates many genes, including those related to organogenesis and lipid metabolism, in the liver during the suckling period. Thus, a maternal AA deficiency in humans, particularly during the lactation period, may exert deleterious effects in babies, which is a crucial issue for lifelong health.

\section{Materials and methods}

Animals. All animal experiments were approved by the Tokyo Medical and Dental University Committee on Animal Research $(\# 0,170,125 \mathrm{~A})$ and the Animal Care and Use Committee of the Tokyo Metropolitan Institute of Gerontology $(\# 17,074)$. All methods involving animals were performed in accordance with the relevant guidelines and regulations. All mice were treated in accordance with the Fundamental Guidelines for the Proper Conduct of Animal Experiments and Related Activities in Academic Research Institutions under the jurisdiction of the Ministry of Education, Culture, Sports, Science and Technology of Japan. All efforts were made to minimize suffering and to reduce the number of animals used.

Pregnant female C57BL6 mice, which were primiparous and housed individually, were purchased from CLEA Japan (Tokyo, Japan) on gestational day 13. After delivery, litter size was adjusted to 5-6 pups (all male) per dam to avoid metabolic drifts due to nutrient availability during the lactation period. SMP30/GNL KO mice were generated using a gene-targeting technique as described previously ${ }^{33}$. Female SMP30/GNL KO (SMP30/ GNL-/-) mice were mated with male SMP30/GNL KO (SMP30/GNLY/-) mice to produce SMP30/GNL KO (male SMP30/GNLY/- and female SMP30/ GNL-/-) mice under normal AA conditions; mice had free access to water containing $1.5 \mathrm{~g} / \mathrm{L} \mathrm{AA}$ and $100 \mu \mathrm{mol} / \mathrm{L}$ EDTA to avoid the effects of an AA deficiency ${ }^{20}$. The concentration of AA in drinking water was sufficient to maintain normal AA levels in all tissues ${ }^{20}$. EDTA was added to stabilize AA in drinking water, which remained in a stable state for at least 4 days ${ }^{20}$. Water bottles were changed every 3 or 4 days. In addition, all mice were fed an AA-free diet (CL-2, CLEA Japan, Tokyo, Japan) ad libitum. During the lactation 
period, KOA and KO dams received an i.p. injection of AA in saline $(4.0 \mathrm{~g} / \mathrm{L})$ and solvent only, respectively. Throughout the experiments, animals were maintained on a 12-h light/dark cycle in a controlled environment.

Measurement of AA concentrations. The total AA concentration, including AA and dehydroascorbic acid (DHA), the oxidized form of AA, was measured using high-performance liquid chromatography and electrochemical detection in accordance with previously described methods ${ }^{34}$.

DNA methylation profiling. Mouse liver genomic DNA was extracted using a standard proteinase K method. MIAMI was performed as described previously ${ }^{19,35}$. Briefly, one microgram of genomic DNA from the samples of two groups was digested with the methylation-sensitive restriction enzyme HpaII followed by adaptor ligation and PCR amplification. Amplified DNA from one sample was labeled with Cy3 and the other with Cy5, and they were then cohybridized to gene promoter arrays containing 41,332 probes. The fluorescence signals obtained were normalized using the global normalization method, and signal differences between samples (i.e. Cy3 signal intensity/Cy5 signal intensity), which indicated DNA methylation differences between samples, were assessed. Genomic DNA was also digested with the methylation-insensitive isoschizomer MspI followed by the same procedure to correct for false positives caused by single nucleotide polymorphisms or incomplete digestion. The HpaII/MspI signal difference for each probe was measured as the methylation difference value. Values of $<0.75$ and $>1.33$ denoted DNAhypomethylation and hypermethylation, respectively. DAVID v6.7 (http:// david.abcc.ncifcrf.gov/) was employed for the pathway analysis. Corrected P-values were used to judge candidate genes $(p<0.05 \text { was considered to be significant })^{22}$.

The complete experimental protocol is available at http://epigenome.dept.showa.gunmau.ac.jp/ hatada/ miami/image/MIAMI\%20Protocol\%20V4.pdf.

Bisulfite-sequencing analysis. A bisulfite-sequencing analysis was performed as follows. To prepare the bisulfite stock solution, $3.8 \mathrm{~g}$ of sodium metabisulfite (\#31,609-45, Nacalai Tesque, Kyoto, Japan), $1.34 \mathrm{~g}$ of ammonium sulfite monohydrate (\#014-03,505, Wako Pure Chemical Industries, Osaka, Japan), and $10 \mathrm{~mL}$ of $50 \%$ ammonium hydrogen sulfite solution (\#014-02,905, Wako) were mixed and heated at $80^{\circ} \mathrm{C}$ until dissolved. To prepare a working bisulfite solution (8.4 M, pH 5.2-5.3), $12 \mathrm{~mL}$ of the bisulfite stock solution was diluted with $2.28 \mathrm{~mL}$ of distilled water $\left(\mathrm{dH}_{2} \mathrm{O}\right)$. Two micrograms of genomic DNA was denatured in $0.3 \mathrm{~N} \mathrm{NaOH}$ in a total volume of $24.75 \mu \mathrm{L}$ at $37^{\circ} \mathrm{C}$ for $30 \mathrm{~min}$. The sample was mixed with $275 \mu \mathrm{L}$ of the working bisulfite solution and incubated at $70^{\circ} \mathrm{C}$ for $1 \mathrm{~h}$ in the dark. DNA was recovered using a genomic DNA purification kit (\#NPK-101, Toyobo Life Science Department, Osaka, Japan) and dissolved in $100 \mu \mathrm{L}$ of $\mathrm{dH}_{2} \mathrm{O}$. The DNA sample was mixed with $100 \mu \mathrm{L}$ of $0.4 \mathrm{~N} \mathrm{NaOH}$ (freshly prepared) and incubated at $37^{\circ} \mathrm{C}$ for $15 \mathrm{~min}$. DNA was recovered by adding $150 \mu \mathrm{L}$ of $5 \mathrm{M}$ ammonium acetate (pH 7.0), $2 \mu \mathrm{L}$ of ethacinmate (\#312-01,791, Nippon Gene, Tokyo, Japan), and $750 \mu \mathrm{L}$ of ethanol. The DNA-containing precipitate was dissolved in $20 \mu \mathrm{L}$ of $10 \mathrm{mM}$ Tris- $\mathrm{HCl} / 1 \mathrm{mM}$ EDTA (TE; pH 7.5) and subjected to PCR amplification. The sequential PCR amplification of Fgf21 was performed using specific primers as follows: forward: TTTAGTTTTTTTTTTTAGATTTAGGAGTGTAGATT, reverse: TCCTCСТCTCAACCTCCATAAAAC. Reaction profiles were 40 cycles at $96{ }^{\circ} \mathrm{C}$ for $15 \mathrm{~s}, 59{ }^{\circ} \mathrm{C}$ for $30 \mathrm{~s}$, and $72{ }^{\circ} \mathrm{C}$ for $60 \mathrm{~s}$. Amplified fragments were ligated into pGEM-T easy vectors (\#A1360, Promega, Madison, WI, USA), and more than 13 clones were sequenced per reaction. We used a web-based quantification tool for the bisulfite-sequencing analysis of CpG methylation (http://quma.cdb.riken.jp/) ${ }^{36}$.

5hmC dot blot analysis. Isolated DNA (450 ng per sample) was denatured in $1 \mathrm{M} \mathrm{NaOH}$ at $95{ }^{\circ} \mathrm{C}$ for $10 \mathrm{~min}$. Samples were neutralized with $5 \mathrm{M} \mathrm{NH}_{4} \mathrm{OAc}$ on ice. DNA samples were spotted on a Nylon membrane (Hybond-N +, GE-Amersham, UK). The blotted membrane was dried at $80^{\circ} \mathrm{C}$ for $30 \mathrm{~min}$, and UV cross-linked at $70,000 \mu$ joules $/ \mathrm{cm}^{2}$. The membrane was then incubated with Blocking One (Nacalai Tesque, Kyoto, Japan) at room temperature for $1 \mathrm{~h}$. The rabbit polyclonal antibody anti-5hmC (No. 39770; Active Motif, USA, dilution 1:5000) in PVDF Blocking Reagent for Can Get Signal 1 (Toyobo, Osaka, Japan) was added at room temperature for $2 \mathrm{~h}$. The membrane was washed for $10 \mathrm{~min} 3 \times$ in TBST buffer, and then incubated with HRP-conjugated anti-rabbit IgG (\#NA934V, GE-Amersham, UK) secondary antibodies in Can Get Signal 2 (Toyobo, Osaka, Japan) at room temperature for $1 \mathrm{~h}$. The membrane was then washed for $10 \mathrm{~min} 3 \times$ in TBST and visualized by chemiluminescence with ECL Prime (GE-Amersham, UK).

Biochemical assays. Serum NEFA, TG, and T-Chol levels were measured using NEFA C-Test Wako (\#27975,401), TG E-Test Wako (\#432-40,210), and T-Chol E-Test Wako (\#439-17,501) kits (Wako Pure Chemical Industries, Ltd., Osaka, Japan), respectively ${ }^{19,22}$. Blood glucose was measured using a glucometer (Glutest PRO R; Sanwa Kagaku Kenkyusho Co., Ltd., Aichi, Japan $)^{22}$. As milk samples, we used the gastric contents of offspring, which mainly consisted of milk derived from dams, on $\mathrm{d} 16^{22}$.

Statistical analysis. Data are expressed as the mean \pm standard error of the mean (SEM). Data were compared using an unpaired Student's $t$-test. Comparisons of body weight differences were performed using a twoway analysis of variance (ANOVA) with Tukey's multiple comparison test. A $p$-value of $<0.05$ was considered to be significant. Statistical analyses were performed using Prism 6 (GraphPad Software, Inc., La Jolla, CA, USA).

\section{Data availability}

Data that support the results of the present study are available from the corresponding author upon reasonable request. 
Received: 13 May 2020; Accepted: 19 November 2020

Published online: 04 December 2020

\section{References}

1. Hackett, J. A. \& Surani, M. A. DNA methylation dynamics during the mammalian life cycle. Philos. Trans. R. Soc. Lond. B Biol. Sci. 368, 20110328. https://doi.org/10.1098/rstb.2011.0328 (2013).

2. Pastor, W. A., Aravind, L. \& Rao, A. TETonic shift: biological roles of TET proteins in DNA demethylation and transcription. Nat. Rev. Mol. Cell. Biol. 14, 341-356. https://doi.org/10.1038/nrm3589 (2013).

3. Seisenberger, S. et al. Reprogramming DNA methylation in the mammalian life cycle: building and breaking epigenetic barriers. Philos. Trans. R. Soc. Lond. B Biol. Sci. 368, 20110330. https://doi.org/10.1098/rstb.2011.0330 (2013).

4. Chung, T. L. et al. Vitamin C promotes widespread yet specific DNA demethylation of the epigenome in human embryonic stem cells. Stem Cells 28, 1848-1855. https://doi.org/10.1002/stem.493 (2010).

5. Esteban, M. A. et al. Vitamin C enhances the generation of mouse and human induced pluripotent stem cells. Cell Stem Cell 6, 71-79. https://doi.org/10.1016/j.stem.2009.12.001 (2010).

6. Blaschke, K. et al. Vitamin C induces Tet-dependent DNA demethylation and a blastocyst-like state in ES cells. Nature 500, $222-226$. https://doi.org/10.1038/nature12362 (2013).

7. Chen, J. et al. Vitamin C modulates TET1 function during somatic cell reprogramming. Nat. Genet. 45, 1504-1509. https://doi. org/10.1038/ng.2807 (2013).

8. Yin, R. et al. Ascorbic acid enhances Tet-mediated 5-methylcytosine oxidation and promotes DNA demethylation in mammals. J. Am. Chem. Soc. 135, 10396-10403. https://doi.org/10.1021/ja4028346 (2013).

9. Starczak, M. et al. In vivo evidence of ascorbate involvement in the generation of epigenetic DNA modifications in leukocytes from patients with colorectal carcinoma, benign adenoma and inflammatory bowel disease. J. Transl. Med. 16, 204. https://doi. org/10.1186/s12967-018-1581-9 (2018).

10. Gillberg, L. et al. Oral vitamin C supplementation to patients with myeloid cancer on azacitidine treatment: Normalization of plasma vitamin C induces epigenetic changes. Clin. Epigenet. 11, 143. https://doi.org/10.1186/s13148-019-0739-5 (2019).

11. Nishikimi, M., Koshizaka, T., Ozawa, T. \& Yagi, K. Occurrence in humans and guinea pigs of the gene related to their missing enzyme L-gulono-gamma-lactone oxidase. Arch. Biochem. Biophys. 267, 842-846. https://doi.org/10.1016/0003-9861(88)90093 -8 (1988).

12. Maruyama, N., Ishigami, A. \& Kondo, Y. Pathophysiological significance of senescence marker protein-30. Geriatr. Gerontol. Int. 10(Suppl 1), S88-98. https://doi.org/10.1111/j.1447-0594.2010.00586.x (2010).

13. Ishigami, A. \& Maruyama, N. Significance of SMP30 in Gerontology. Geriatr. Gerontol. Int. 7, 316-325. https://doi.org/10.111 $1 / \mathrm{j} .1447-0594.2007 .00420 . \mathrm{x}(2007)$

14. Kondo, Y. et al. Senescence marker protein 30 functions as gluconolactonase in L-ascorbic acid biosynthesis, and its knockout mice are prone to scurvy. Proc. Natl. Acad. Sci. USA 103, 5723-5728. https://doi.org/10.1073/pnas.0511225103 (2006).

15. Kishimoto, Y. et al. Insufficient ascorbic acid intake during gestation induces abnormal cardiac dilation in fetal and neonatal SMP30/GNL knockout mice. Pediatr. Res. 73, 578-584. https://doi.org/10.1038/pr.2013.22 (2013).

16. Schjoldager, J. G. et al. Maternal vitamin C deficiency during pregnancy results in transient fetal and placental growth retardation in guinea pigs. Eur. J. Nutr. 54, 667-676. https://doi.org/10.1007/s00394-014-0809-6 (2015).

17. Hansen, S. N., Tveden-Nyborg, P. \& Lykkesfeldt, J. Does vitamin C deficiency affect cognitive development and function?. Nutrients 6, 3818-3846. https://doi.org/10.3390/nu6093818 (2014).

18. Shorey-Kendrick, L. E. et al. Vitamin C prevents offspring DNA methylation changes associated with maternal smoking in pregnancy. Am. J. Respir. Crit. Care Med. 196, 745-755. https://doi.org/10.1164/rccm.201610-2141OC (2017).

19. Ehara, T. et al. Ligand-activated PPAR $\alpha$-dependent DNA demethylation regulates the fatty acid $\beta$-oxidation genes in the postnatal liver. Diabetes 64, 775-784. https://doi.org/10.2337/db14-0158 (2015).

20. Iwama, M., Shimokado, K., Maruyama, N. \& Ishigami, A. Time course of vitamin C distribution and absorption after oral administration in SMP30/GNL knockout mice. Nutrition 27, 471-478. https://doi.org/10.1016/j.nut.2010.04.010 (2011).

21. Hatada, I. et al. Genome-wide demethylation during neural differentiation of P19 embryonal carcinoma cells. J. Hum. Genet. 53, 185-191. https://doi.org/10.1007/s10038-007-0228-0 (2008).

22. Yuan, X. et al. Epigenetic modulation of Fgf21 in the perinatal mouse liver ameliorates diet-induced obesity in adulthood. Nat. Commun. 9, 636. https://doi.org/10.1038/s41467-018-03038-w (2018).

23. Inagaki, T. et al. Endocrine regulation of the fasting response by PPARalpha-mediated induction of fibroblast growth factor 21 . Cell Metab. 5, 415-425. https://doi.org/10.1016/j.cmet.2007.05.003 (2007).

24. Amano, A., Aigaki, T., Maruyama, N. \& Ishigami, A. Ascorbic acid depletion enhances expression of the sodium-dependent vitamin C transporters, SVCT1 and SVCT2, and uptake of ascorbic acid in livers of SMP30/GNL knockout mice. Arch. Biochem. Biophys. 496, 38-44. https://doi.org/10.1016/j.abb.2010.01.012 (2010).

25. Ishigami, A. et al. SMP30 deficiency in mice causes an accumulation of neutral lipids and phospholipids in the liver and shortens the life span. Biochem. Biophys. Res. Commun. 315, 575-580. https://doi.org/10.1016/j.bbrc.2004.01.091 (2004).

26. Ha, T. Y., Otsuka, M. \& Arakawa, N. The effect of graded doses of ascorbic acid on the tissue carnitine and plasma lipid concentrations. J. Nutr. Sci. Vitaminol. (Tokyo) 36, 227-234. https://doi.org/10.3177/jnsv.36.227 (1990).

27. DiTroia, S. P. et al. Maternal vitamin C regulates reprogramming of DNA methylation and germline development. Nature 573, 271-275. https://doi.org/10.1038/s41586-019-1536-1 (2019).

28. Maeda, N. et al. Aortic wall damage in mice unable to synthesize ascorbic acid. Proc. Natl. Acad. Sci. USA 97, 841-846. https:// doi.org/10.1073/pnas.97.2.841 (2000).

29. Hill, P. W. S. et al. Epigenetic reprogramming enables the transition from primordial germ cell to gonocyte. Nature 555, 392-396. https://doi.org/10.1038/nature25964 (2018).

30. Thomassin, H., Flavin, M., Espinás, M. L. \& Grange, T. Glucocorticoid-induced DNA demethylation and gene memory during development. EMBO J. 20, 1974-1983. https://doi.org/10.1093/emboj/20.8.1974 (2001).

31. Amenya, H. Z., Tohyama, C. \& Ohsako, S. Dioxin induces Ahr-dependent robust DNA demethylation of the Cyplal promoter via Tdg in the mouse liver. Sci. Rep. 6, 34989. https://doi.org/10.1038/srep34989 (2016).

32. Pan, X. et al. Methylation of the Corticotropin Releasing Hormone Gene Promoter in BeWo Cells: Relationship to Gene Activity. Int. J. Endocrinol. 2015, 861302. https://doi.org/10.1155/2015/861302 (2015).

33. Ishigami, A. et al. Senescence marker protein-30 knockout mouse liver ishighly susceptible to tumor necrosis factor-alpha- and Fas-mediated apoptosis. Am. J. Pathol. 161, 1273-1281. https://doi.org/10.1016/s0002-9440(10)64404-5 (2002).

34. Sato, Y. et al. Determination of dehydroascorbic acid in mouse tissues and plasma by using tris(2-carboxyethyl)phosphine hydrochloride as reductant in metaphosphoric acid/ethylenediaminetetraacetic acid solution. Biol. Pharm. Bull. 33, 364-369. https:// doi.org/10.1248/bpb.33.364 (2010).

35. Kobayashi, Y. et al. DNA methylation changes between relapse and remission of minimal change nephrotic syndrome. Pediatr. Nephrol. 27, 2233-2241. https://doi.org/10.1007/s00467-012-2248-z (2012).

36. Kumaki, Y., Oda, M. \& Okano, M. QUMA: quantification tool for methylation analysis. Nucleic Acids Res. 36, W170-175. https:// doi.org/10.1093/nar/gkn294 (2008). 


\section{Acknowledgments}

This work was supported in part by Grants-in-Aid for Scientific Research (KAKENHI) from the Japan Society for the Promotion of Science (JSPS) [grant Numbers 19K09018 (K.H.), 16H05331 (Y.O.)]; Secom Science and Technology Foundation (Y.O.); Takeda Science Foundation (K.H.); and Mishima Kaiun Memorial Foundation (K.H.).

\section{Author contributions}

K.H. conceived the project, designed the experiments, and evaluated the data; K.K, performed the experiments and prepared figures and tables, X.Y., N.H., K.T., and F.W. participated in and contributed to all experiments; Y.Ka. and T.K. contributed to the 5hmC dot blot analysis; A.I. and Y.Ko. performed experiments using SMP30/ GNL KO mice; T.Y. evaluated the data; K.H. and Y.O. wrote the manuscript and supervised the entire project. All authors discussed the results, commented on the manuscript, and gave final approval of the version to be submitted.

\section{Competing interests}

The authors declare no competing interests.

\section{Additional information}

Supplementary information is available for this paper at https://doi.org/10.1038/s41598-020-77962-7.

Correspondence and requests for materials should be addressed to Y.O. or K.H.

Reprints and permissions information is available at www.nature.com/reprints.

Publisher's note Springer Nature remains neutral with regard to jurisdictional claims in published maps and institutional affiliations.

(c) (i) Open Access This article is licensed under a Creative Commons Attribution 4.0 International License, which permits use, sharing, adaptation, distribution and reproduction in any medium or format, as long as you give appropriate credit to the original author(s) and the source, provide a link to the Creative Commons licence, and indicate if changes were made. The images or other third party material in this article are included in the article's Creative Commons licence, unless indicated otherwise in a credit line to the material. If material is not included in the article's Creative Commons licence and your intended use is not permitted by statutory regulation or exceeds the permitted use, you will need to obtain permission directly from the copyright holder. To view a copy of this licence, visit http://creativecommons.org/licenses/by/4.0/.

(c) The Author(s) 2020 